\title{
A Percepção de uma Equipe sobre o Processo de Gerência de Configuração em Relação ao MPS.BR Nível F: Um Estudo de Caso
}

\author{
Relato de Experiência \\ José Jorge L. Dias Jr., Arkjoaquitonyo E. da Silva, Fernanda N. M. Dias \\ ${ }^{1}$ Centro de Ciências Aplicadas e Educação - Universidade Federal Paraíba (UFPB) \\ Rio Tinto - PB - Brasil \\ \{jorge, tony.silva\}@dce.ufpb.br, fernanda.nmdias@gmail.com
}

\begin{abstract}
The configuration management is one of the required processes to achieve the level F of MPS.BR model. Companies that aims at desiring to achieve this maturity level must define and control the process in order to use it in an effective way. However, it is important to understand the team perceptions related to process using. In this sense, this work presents a case study, supported by a qualitative methodology, to analyze the team perceptions related to expected results of configuration management of MPS.BR model and to verify if these perceptions are reflected on the audit results.
\end{abstract}

Resumo. A gerência de configuração (GCO) é um dos processos exigidos para se atingir a maturidade do nivel $F$ do MPS.BR. Empresas que desejam atingir este nivel de maturidade definem o processo e o controlam para que esse seja utilizado de maneira efetiva. Entretanto, é importante entender as percepções da equipe em relação ao uso do processo. Neste sentido, este trabalho apresenta um estudo de caso, apoiado por metodologia qualitativa, para analisar as percepções de uma equipe em relação aos resultados esperados do processo de GCO do MPS.BR e verificar se estas percepções refletem os resultados das auditorias realizadas.

\section{Introdução}

Cada vez mais a engenharia de software está sendo investigada sobre os pilares da ciência e pra isso os métodos científicos adequados precisam ser utilizados para que surjam teorias fortes e confiáveis. A metodologia qualitativa é uma opção para aqueles que desejam obter uma análise mais analítica e rica, principalmente relacionados aos aspectos humanos. Alguns exemplos de trabalhos nesta ótica é o de Menolli (2011), onde foi realizado um estudo exploratório sobre as percepções dos gerentes de projeto em relação às práticas do modelo MPS em Fábricas de Software no Brasil. Outro exemplo é o de Dos Santos et al. (2011), onde foram discutidos os principais resultados de uma pesquisa qualitativa para analisar quais fatores humanos tiveram maior influência do ponto de vista dos colaboradores das organizações.

Empresas têm investido esforços para atingir níveis de maturidade em relação a modelos como MPS.BR [Softex 2012] para se tornarem mais competitivas no mercado de desenvolvimento de software. Para isso, a empresa precisa se preparar e cumprir os resultados esperados dos processos exigidos do nível o qual se espera alcançar. Mais do 
que isso, os elementos cobrados pelo MPS.BR devem ser tratados com naturalidade e trazer benefícios reais à empresa. Um dos processos exigidos é o de Gerência de Configuração (GCO), presente no nível F do MPS.BR. Segundo este modelo, sete resultados esperados precisam ser satisfeitos em relação a este processo.

As organizações enfrentam uma grande dificuldade em adotar conceitos e práticas de GCO, pois as atividades envolvidas neste processo são complexas, envolvem a definição de uma abordagem adequada e a seleção de ferramentas de apoio as suas atividades [Fernandes 2011].

O processo de GCO contribui para a melhoria da qualidade sendo fortemente focada em controle. Uma das maneiras de realizar este controle é através de auditorias, porém, a percepção da equipe envolvida no desenvolvimento do software pode apresentar resultados diferentes do que normalmente é apresentado por estas auditorias. Partindo desta hipótese, foi definida a seguinte questão para orientar a pesquisa: "Qual a percepção da equipe em relação aos Resultados Esperados do processo de Gerência de Configuração do MPS.BR Nivel F?".

Para responder a esta questão foi realizada uma pesquisa qualitativa através de um estudo de caso seguindo as orientações de Yin (2010). Em relação a análise dos dados, foram utilizadas técnicas da Grounded Theory (teoria fundamentada dos dados) com base em Strauss \& Corbin (1990). As percepções identificadas foram relacionadas aos sete resultados esperados do MPS.BR. Além disso, foi analisado o gap entre as percepções e alguns resultados de auditorias realizadas na empresa.

Esse artigo está organizado da seguinte forma: a Seção 2 apresenta a metodologia utilizada para realização do estudo de caso. A Seção 3 discute os resultados da análise apresentando as percepções identificadas. A Seção 4 apresenta uma discussão sobre a relação das percepções com os resultados esperados do processo de GCO do MPS.BR e com os resultados das auditorias, e discute algumas lições aprendidas. Finalmente, a Seção 5 discorre sobre as considerações finais.

\section{Metodologia}

Para a realização desta pesquisa, foi seguido um conjunto de cinco etapas de acordo com a Figura 1.

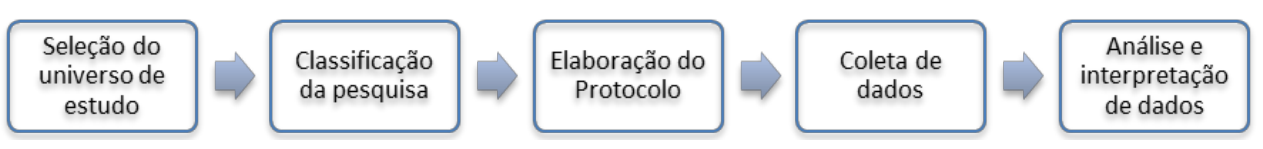

Figure 1. Etapas da Pesquisa

As próximas subseções explicam em detalhes cada uma das fases da metodologia adotada.

\subsection{Seleção do Universo de Estudo}

Esta pesquisa foi realizada junto à uma equipe de uma empresa de software que é composta por 01 Gerente de Projetos/Scrum Master, 01 Gerente de Configuração, 01 Product Owner e 04 desenvolvedores. Atualmente a empresa está em processo de aderência ao MPS.BR nível $\mathrm{F}$ e por isso tem passado por constantes mudanças em sua metodologia, além da adaptação da equipe para ficar em conformidade com todos os processos exigidos por este nível. 


\subsection{Classificação da Pesquisa}

Esta pesquisa qualifica-se como um estudo de caso único, pois está limitado à realidade de um único grupo de pessoas e realizado de forma a analisar com maior profundidade o processo de GCO como disciplina para atingir o nível de maturidade F do MPS.BR.

Em relação à natureza, esta pesquisa é aplicada e tem como objetivo gerar conhecimentos para aplicação prática, dirigidos à solução de problemas específicos. Além disso, esta pesquisa tem o propósito descritivo, por descrever os fatos e fenômenos de determinada realidade expondo as características da organização na visão dos pesquisadores e das pessoas que a compõem [Triviños 1987].

\subsection{Elaboração do Protocolo}

Este estudo foi guiado por um protocolo, que é um instrumento de orientação para os pesquisadores durante a realização da pesquisa, uma vez que apresenta informações sobre a base teórica que sustenta o estudo. Também neste protocolo foram definidos os procedimentos e instrumentos para a coleta e análise dos dados. O protocolo completo pode ser solicitado diretamente aos autores deste trabalho.

\subsection{Coleta de dados}

A coleta de dados é uma fase essencial na pesquisa, pois evidencia os fatos e as hipóteses encontradas no estudo. Assim, alguns princípios foram adotados para manter a qualidade do estudo de caso: entrevistas semiestruturadas, análise documental e observação direta/participante.

Neste sentido, foram elaborados dois roteiros de entrevista, um para os funcionários com cargos técnicos e outro para cargos gerenciais. Apesar de possuírem perguntas distintas, abordavam o mesmo conteúdo. A diferenciação entre os dois questionários se deu de acordo com o conhecimento exigido pelo MPS.BR para cada nível na hierarquia de funcionários. Foram realizadas cinco entrevistas, totalizando aproximadamente 35 minutos e 27 segundos de áudio relacionadas apenas as respostas e não as perguntas do entrevistador.

A análise documental foi utilizada para auxiliar na corroboração das evidências. Foram considerados evidências para esta fonte documentos/artefatos utilizados pela GCO e armazenados nos repositórios da organização.

As observações diretas informais foram realizadas atendo-se apenas a comportamentos e fatos relacionados à GCO sem a necessidade de desenvolvimento de instrumentos observacionais no protocolo de estudo. Já a observação participante, sendo uma modalidade especial de observação em que o observador participa dos eventos estudados, permitiu o pesquisador ter acesso a eventos, de outro modo inacessível, e ainda a pontos de vista interno ao estudo de caso.

\subsection{Análise dos dados}

Com os dados coletados, os resultados foram gerados utilizando técnicas de grounded theory ou teoria fundamentada dos dados [Strauss \& Corbin 1990]. Este é um método de pesquisa qualitativo que utiliza procedimentos sistemáticos para gerar teoria através de codificação. O processo foi dividido em quatro fases, sendo elas: Identificação do trecho; Rotulagem; Categorização; e Geração de hipóteses. 
Após a transcrição dos áudios, realizamos a quebra dos textos, examinamos e elaboramos os rótulos. Em seguida, com os conceitos mais claros, foi possível elaborar categorias para agrupar rótulos que tivessem proposições com a mesma ideia da categoria. Por fim, as relações entre categorias foram mapeadas, onde foram realizadas a elaboração de hipóteses.

Após a definição de todos os rótulos foi necessário um refinamento destes para melhor refletir as características dos trechos identificados. Para isto os trechos foram examinados e relidos inúmeras vezes. Em seguida, foram realizadas algumas composições para rótulos que correspondiam a mesma ideia.

Desta forma, os dados foram transformados em rótulos e agrupados de acordo com sua semelhança de conteúdo. A partir disto, foram classificados em categorias. Ao final, foram gerados 12 rótulos organizados em 6 categorias diferentes.

\section{Resultados}

$\mathrm{Na}$ fase de análise e interpretação dos dados foram levantadas 12 hipóteses referentes a cada um dos rótulos agrupados em cinco categorias: Diretrizes; Controle; Apoio, Conhecimento; Adaptação; e Aderência.

Nesta seção discutiremos os resultados de cada categoria emergida através da análise qualitativa.

\subsection{Diretrizes}

Para a categoria Diretrizes foram levantadas, com base nas evidências, duas hipóteses: O Plano de Gerência de Configuração é conhecido pela equipe de software, mas não é sabido seu exato conteúdo; e O plano de Gerência de Configuração quando bem definido faz com que a equipe não tenha a necessidade de adquirir o conhecimento detalhado sobre a execução das atividades do processo de GCO.

Apesar da equipe de software saber da existência do Plano de GCO e ter acesso a este, o seu conteúdo era conhecido apenas em partes. O Gerente de Configuração era o único que conhecia exatamente o que havia no documento. Porém, mesmo não sabendo o seu conteúdo completamente, a equipe sabia que o processo de GCO exigido pela empresa estava todo definido no plano. Desta forma não sentiam necessidade em conhecer melhor e absorver novos conhecimentos referente as atividades da GCO. Ao conhecer mais a fundo e adquirir novos conhecimentos, ao invés de simplesmente executar os procedimentos, as atividades assim como todo o processo de GCO poderiam ser melhoradas.

\subsection{Controle}

$\mathrm{Na}$ categoria Controle também foram levantadas duas hipóteses: A atividade de controle dos itens de configuração não é entendida; e a utilidade da baseline deve ser melhor entendida pela equipe de software.

Mesmo o controle dos itens de configuração sendo uma das atividades essenciais no processo de GCO, esta não era bem entendida pela equipe. Quando perguntados sobre o controle, os entrevistados ficavam muito confusos em relação a como realmente a atividade era realizada. Existe até o risco de estar sendo executada uma atividade de forma errada ou talvez ineficiente. Algum problema, seja qual for, em qualquer parte do 
processo de $\mathrm{GCO}$, pode acarretar em problemas maiores em algum momento no desenvolvimento do software. Então é importante ter o entendimento sobre cada uma das atividades, principalmente por aqueles que irão executá-las. Quando se falava em baseline poucos sabiam sua finalidade mesmo as utilizando em vários momentos. Sabendo a utilidade de uma baseline, certamente os itens que a geram serão produzidos sob uma melhor perspectiva.

\subsection{Apoio}

Para a categoria Apoio só foi levantada uma hipótese: A equipe de software utiliza ferramentas de suporte na realização de atividades do processo de GCO.

Todas as ferramentas de suporte ao processo de GCO adotadas pela empresa são utilizadas pela equipe de software. As ferramentas podem ajudar a aumentar a produtividade, organizar as atividades e fornecer características essenciais em um processo de GCO, como por exemplo, o controle de versão e o controle de mudanças. Seria interessante focar em um conhecimento mais aprimorado das ferramentas utilizadas, oferecendo assim maior confiança na utilização das ferramentas e nas resoluções de possíveis problemas.

\subsection{Conhecimento}

Foram levantadas três hipóteses para a categoria Conhecimento: Não existe o entendimento sobre os conceitos envolvidos no processo de GCO; Não existe o entendimento necessário sobre as atividades envolvidas no processo de GCO; e Insegurança da equipe em relação ao conhecimento das atividades realizadas no processo de GCO.

Em toda a pesquisa foi percebida a falta de conhecimento por parte da equipe em muitos momentos do processo de GCO, apesar do processo está sendo executado e a auditoria estar satisfeita com os resultados obtidos. O interesse pelo conhecimento deve ser estimulado dentro da organização e é importante tanto para o processo de GCO quanto para os demais processos existentes no desenvolvimento de software. É necessário o nivelamento do conhecimento da equipe, procurando sempre evitar a centralização deste. Através do conhecimento adequado, as atividades são executadas com maior segurança e naturalidade. Além disso, as resoluções de problemas ficam mais fáceis e o processo ganha mais qualidade.

\subsection{Adaptação}

Para a categoria Adaptação também foram levantados três hipóteses: A equipe teve dificuldades para se adaptar as atividades do processo de GCO; A equipe passou por diversas mudanças quanto a implantação do processo de GCO; É percebido pela equipe que existem muitos procedimentos a serem seguidos.

A adaptação ao processo de GCO foi o maior problema relatado pela equipe de software, pois muitas mudanças foram feitas ao longo da implantação. Por exemplo, houve a mudança do Gerente de Configuração, a entrada e saída de desenvolvedores e a equipe de consultores não se manteve no decorrer da implantação do processo. Estes e outros fatores, como a falta de conhecimento sobre o processo de GCO, acabaram dificultando o entendimento e talvez a aceitação do processo de GCO por completo. Devido às mudanças, novos procedimentos foram estabelecidos no processo ou foram 
modificados. É importante manter a atenção quanto as diversas mudanças sempre procurando analisar seus impactos.

\subsection{Aderência}

Já para a categoria Aderência foi levantada apenas uma hipótese: A auditoria é percebida e realizada pela equipe de software.

Toda a equipe estava ciente das auditorias que eram realizadas referentes ao processo de GCO. O acompanhamento que a auditoria realiza para verificar se todos as atividades esperadas estão sendo executados é fundamental para manter um certo controle sobre a equipe e também sobre o processo. É interessante aliar a auditoria a outros procedimentos a fim de não só acompanhar as atividades do processo, mas tentar gerar uma análise completa sobre o andamento da GCO.

\section{Discussão}

\subsection{Percepções e o Processo de GCO do MPS.BR}

A Tabela 1 mostra uma matriz gerada relacionando as categorias que emergiram na análise com os resultados esperados pelo processo de GCO do MPS.BR nível F. A tabela sintetiza quais foram as percepções da equipe para cada resultado esperado. Para cada resultado esperado pode se analisar quais foram as percepções negativas identificadas que precisam de uma maior atenção. Isto quer dizer que o conhecimento sobre as hipóteses levantadas para cada uma das categorias permite às organizações tomarem decisões/ações de melhorias referente à implantação do processo de GCO.

Tabela 1. Relação das categorias com os resultados esperados

\begin{tabular}{|l|c|c|c|c|c|c|c|}
\hline \multirow{2}{*}{$\begin{array}{l}\text { Categorias das } \\
\text { percepções }\end{array}$} & \multicolumn{6}{|c|}{ Resultados esperados pelo processo de GCO do MPS.BR } \\
\cline { 2 - 8 } & GCO 1 & GCO 2 & GCO 3 & GCO 4 & GCO 5 & GCO 6 & GCO 7 \\
\hline Diretrizes & $\mathrm{X}$ & $\mathrm{X}$ & $\mathrm{X}$ & & & & \\
\hline Controle & & & $\mathrm{X}$ & $\mathrm{X}$ & $\mathrm{X}$ & $\mathrm{X}$ & \\
\hline Apoio & $\mathrm{X}$ & $\mathrm{X}$ & $\mathrm{X}$ & $\mathrm{X}$ & $\mathrm{X}$ & $\mathrm{X}$ & \\
\hline Conhecimento & $\mathrm{X}$ & $\mathrm{X}$ & $\mathrm{X}$ & & & & \\
\hline Adaptação & $\mathrm{X}$ & $\mathrm{X}$ & $\mathrm{X}$ & $\mathrm{X}$ & $\mathrm{X}$ & $\mathrm{X}$ & \\
\hline Aderência & & & & & & & $\mathrm{X}$ \\
\hline
\end{tabular}

\subsection{Confrontando as auditorias com as perceções identificadas}

A auditoria realizada na empresa confere os itens de um checklist contendo questionamentos que vão de encontro ao que se espera da GCO conforme exigido pelo MPS.BR nível F. O objetivo é analisar os índices e assim tomar atitudes de melhorias ou correções para o processo. Porém a auditoria realizada cobre apenas algumas das atividades da GCO, como pode ser visto na Tabela 2.

Tabela 2. Auditorias de planejamento e encerramento

\begin{tabular}{|c|c|c|c|}
\hline & Item & Situação & $\begin{array}{c}\text { Percentual de } \\
\text { aderência }\end{array}$ \\
\hline & As baselines do projeto foram planejadas? & Conforme & \multirow{2}{*}{$100 \%$} \\
\hline$\pi \cdot 7$ & A baseline de planejamento foi gerada? & Conforme & \\
\hline
\end{tabular}




\begin{tabular}{|c|c|c|}
\hline $\begin{array}{l}\text { Os itens previstos para a baseline de planejamento foram } \\
\text { definidos (Plano de Projeto, Plano de Escopo)? }\end{array}$ & Conforme & \\
\hline As versões dos itens estão adequadas? & Conforme & \\
\hline $\begin{array}{l}\text { Os itens de configuração estão definidos conforme o } \\
\text { padrão? }\end{array}$ & Conforme & \\
\hline A baseline de encerramento do projeto foi definida? & Conforme & \multirow{7}{*}{$100 \%$} \\
\hline $\begin{array}{l}\text { Os itens previstos para a baseline de encerramento foram } \\
\text { definidos (Plano de Projeto, Plano de Escopo, Planilha de } \\
\text { Monitoramento e controle, Planilha de Indicadores, e } \\
\text { Termo de Encerramento do projeto)? }\end{array}$ & Conforme & \\
\hline As versões dos itens estão adequadas? & Conforme & \\
\hline Os itens de configuração estão completos? & Conforme & \\
\hline $\begin{array}{l}\text { Os itens previstos para a baseline de encerramento foram } \\
\text { definidos (Plano de Projeto, Plano de Escopo, Planilha de } \\
\text { Monitoramento e controle, Planilha de Indicadores, e } \\
\text { Termo de Encerramento do projeto)? }\end{array}$ & Conforme & \\
\hline As versões dos itens estão adequadas? & Conforme & \\
\hline Os itens de configuração estão completos? & Conforme & \\
\hline
\end{tabular}

Na Tabela 2 são apresentados dados referentes a auditoria realizada no mês de abril. Na coluna "Percentual de aderência" podemos ver os percentuais de aderência ao processo de GCO implantado na empresa. Os percentuais são calculados da seguinte maneira: totais de itens multiplicado por 100 dividido pelo número de itens conformes.

Com base nestes dados são gerados relatórios, os quais servem para que a diretoria da empresa tome ciência da aderência ao processo de GCO. Esses relatórios descrevem um breve diagnóstico sobre os resultados de cada sprint baseados nas auditorias de planejamento e encerramento.

Ocorre que a auditoria usa como base para a realização de suas análises um entendimento próprio dos fenômenos e fatos ocorridos no processo de GCO, usando os percentuais de aderência apenas como gatilhos para tomadas de ações, sejam de melhorias quando são percentuais aceitáveis, sejam de correções quando não são aceitáveis pela empresa.

Além do mês de abril foram analisados os resultados das auditorias dos meses de janeiro e março para confrontar com as percepções analisadas neste trabalho. A conformidade nestes meses foram $20 \%$ e $100 \%$ no mês de março, e $80 \%$ e $100 \%$ no mês de janeiro. Pudemos perceber que a aderência alcançou seu percentual mais alto em vários momentos, exceto em dois que foram abaixo dos $100 \%$. Contudo esta pesquisa mostra um processo de GCO que não é entendido por completo nem executado com eficiência pela equipe de software. Para tanto, as hipóteses levantadas neste estudo podem auxiliar a tomadas de decisões em conjunto com as auditorias já realizadas.

\subsection{Lições Aprendidas}

Este trabalho permitiu perceber a importância da aplicação de uma metodologia qualitativa, executada com rigor científico, para analisar as percepções de equipes de softwares relacionadas aos processos. Com isso, foi identificado que a percepção puramente baseada no senso comum, sem aplicação de análises, pode ser interpretada erroneamente, principalmente para os gestores, trazendo a conclusão errada de que o processo está bem definido e está sendo utilizado de maneira efetiva. Muitas vezes as auditorias de qualidade podem resultar em conformidades com o processo, no entanto, a 
percepção e o entendimento da equipe pode ser diferente, como foi mostrado neste trabalho.

A comunicação desempenha um papel fundamental na execução do processo de GCO e deve ser suficientemente abrangente. O compartilhamento de informações e disseminação do conhecimento devem existir para que todos os envolvidos possam ser atingidos.

Nem sempre os instrumentos utilizados pela auditoria para avaliar o andamento da GCO irão corresponder a realidade ocorrida no ambiente onde o processo está implantado, pois estes analisam apenas se as atividades estão sendo realizadas independente se as atividades que levam ao resultado esperado esteja correta ou não.

\section{Considerações Finais}

Este estudo possibilitou a identificação de diversos fatores que permitiram entender melhor a percepção da equipe de software de uma empresa referente ao processo de Gerência de Configuração em relação aos resultados esperados da GCO do MPS.BR. A partir destas percepções podem ser tomadas medidas a fim de que o processo seja melhor entendido e realizado, assim como adequado a realidade e necessidades da empresa.

Assim, concluímos que este estudo contribui para a área de Gerência de Configuração de Software, servindo como um ponto de vista diferenciado, pois leva em consideração a percepção da equipe de software de um projeto para alcançar o nível $\mathrm{F}$ do MPS.BR. e servindo também como uma importante contribuição para o aumento do rigor cientifico na área da Engenharia de Software.

\section{Referências}

Fernandes, J. M. (2011). "Metodologia para a Implantação da Gerência de Configuração de Software em Empresas de Médio Porte". Rio de Janeiro, 130p. Universidade Estadual do Ceará, Mestrado Profissional em Computação Aplicada.

Strauss, A., Corbin, J. (1998). "Basics of Qualitative Research: Techniques and Procedures for Developing Grounded Theory". 2 ed. London, SAGE Publications.

Triviños, A. N. S. (1987). "Introdução à pesquisa em ciências sociais: a pesquisa qualitativa em educação". São Paulo: Atlas.

Yin, R. K. (2010) "Estudo de caso: planejamento e métodos" 4.ed. Porto Alegre: Bookman, 248p; $23 \mathrm{~cm}$.

SOFTEX (2012). "Melhoria de Processo do Software Brasileiro, Guia Geral". Softex. Disponível em: http://www.softex.br/wpcontent/uploads/2013/07/MPS.BR_Guia_Geral_Software_20121.pdf.

Menolli, A. et al. (2011). "Práticas do Modelo MPS em Fábricas de Software: um estudo exploratório sobre as percepções dos gerentes de projeto". In: Simpósio Brasileiro de Qualidade de Software, 2011, Curitiba.

Dos Santos, V. et al. (2011). "Programas de Melhoria de Processo de Software - Uma pesquisa sobre a influência dos aspectos humanos". In: Simpósio Brasileiro de Qualidade de Software, Curitiba. 